\title{
Performance of three- and five-stack achromatic half-wave plates at millimeter wavelengths
}

\author{
Tomotake Matsumura, ${ }^{1,2, \star}$ Shaul Hanany, ${ }^{1}$ Peter Ade, ${ }^{3}$ Bradley R. Johnson, ${ }^{4}$ \\ Terry J. Jones, ${ }^{1}$ Prashanth Jonnalagadda, ${ }^{5}$ and Giorgio Savini ${ }^{6}$ \\ ${ }^{1}$ School of Physics and Astronomy, University of Minnesota, Twin Cities, 116 Church Street SE, \\ Minneapolis, Minnesota 55455, USA
}
${ }^{2}$ Currently with California Institute of Technology, 1200 East California Boulevard, Mail Code 367-17, Pasadena, California 91125, USA
${ }^{3}$ School of Physics and Astronomy, Cardiff University, The Parade, Cardiff CF24 3AA, Wales, UK
${ }^{4}$ Department of Astrophysics University of Oxford, Keble Road, Oxford OX1 3RH, England, UK
${ }^{5}$ Department of Computer Science and Engineering, University of Minnesota,
Twin Cities, 200 Union Street SE, Minneapolis, Minnesota 55455, USA
${ }^{6}$ Optical Science Laboratory, Department of Physics and Astronomy, University College London, Gower Street, London WC1E 6BT, UK
*Corresponding author: tm@caltech.edu

Received 20 April 2009; accepted 30 April 2009;

posted 19 May 2009 (Doc. ID 110202); published 22 June 2009

\begin{abstract}
We study the performance of achromatic half-wave plates (AHWPs) as a function of the detection bandwidth of a power detector operating in the millimeter wave band and the spectral shape of the incident radiation. We focus particular attention on the extraction of the degree of incident polarization and its orientation angle from the intensity measured as a function of AHWP rotation angle, which we call the IVA (intensity versus angle). We describe the formalism to extract the two incident polarization parameters. We use this formalism to quantify the phase offset of the IVA and point to potential systematic errors in the extraction of this offset in cases where the incident spectrum is not sufficiently well known. We quantify the phase offset and modulation efficiency as a function of the relative angles between the plates in the stack and find that high modulation efficiency can be achieved with alignment accuracy of a few degrees. We present measurements of the spectral response of an AHWP made with five plates. The measurements predict a modulation efficiency that is higher than $98 \%$ for three bands centered at 150 , 250, and $410 \mathrm{GHz}$. (C) 2009 Optical Society of America

OCIS codes: $120.5410,230.4110$.
\end{abstract}

\section{Introduction}

Recent experimental efforts in observational cosmology have been focused on searching for a signature from an inflationary period that occurred a short instant after the big bang. This signature is predicted to be imprinted in the polarization of the cosmic

0003-6935/09/193614-12\$15.00/0

(C) 2009 Optical Society of America microwave background (CMB) radiation. Inflation predicts an inflationary gravitational-wave background (IGB) that left a particular pattern of linear polarization on the CMB. This pattern is different from the stronger pattern originating from primordial density anisotropy, which is the main source for the spatial intensity fluctuations over the sky. In the past few years, several groups have started to characterize the polarization signal coming from the primordial density anisotropy $[1,2]$. The 
polarization pattern from the IGB is expected to be at least an order of magnitude smaller, and it has not been detected yet.

Thorough understanding of foregrounds and good control of systematic errors will be required to extract the small signal from the IGB. Both requirements lead to polarimeter designs that implement broad frequency coverage. Examples of such polarimeters are EBEX, a NASA supported balloonborne experiment [3] that is being constructed by the authors of this paper and their collaborators, BICEPII, CLOVER, Keck, PAPPA, PolarBear, QUIET, and SPIDER [4-9].

A common technique to measure linearly polarized radiation is to use a rotating half-wave plate (HWP) together with a linear polarizer. The technique has been used extensively in the optical and IR wavelengths [10-13]. The first experiment to report CMB polarization results with this technique was MAXIPOL [14,15]. Although a HWP is a device that operates over a narrow band of frequencies, there are standard techniques to construct an "achromatic HWP" (AHWP) that operates over a much broader range [16-22]. An AHWP is a stack of birefringent plates that are aligned with specific relative orientation angles between their optic axes. With an appropriate choice of angles, it is possible to achieve modulation efficiency that is close to $100 \%$ over a large fraction of the millimeter wavelength band [20].

The linear polarization content of incident radiation can be characterized in terms of two parameters, the degree of polarization $P_{\text {in }}$ and the orientation angle $\alpha_{\text {in }}$. An equivalent set is the normalized Stokes parameters $Q_{\text {in }} / I_{\text {in }}$ and $U_{\text {in }} / I_{\text {in }}$ (see Section 2 for a definition). To reconstruct these parameters from the signal detected by the instrument, it is essential to quantify the extent to which the polarimeter itself changes the input parameters.

In this paper we study the effects introduced by three- and five-stack AHWPs that are designed to fit CMB polarimeters operating in the range 120 $480 \mathrm{GHz}$. This work is partially motivated by EBEX and a number of other CMB experiments that are either planning to use or considering the use of HWPs and AHWPs to search for faint signals from the IGB. In Sections 2 and 3 we describe the mathematical formalism and define the figures of merit that are used to reconstruct the state of incident polarized light from the measured intensity. In Section 4 we use the figures of merit to quantify how well the incident polarization can be reconstructed. Section 5 discusses effects that arise from uncertainties in the spectrum of the incident radiation. In Section 6 we assess the performance of an AHWP as a function of its construction parameters, and in Section 7 we report on transmission spectra of a five-stack $A \bar{H} W P$ that was constructed for the EBEX experiment. A summary of the key conclusions is given in Section $\underline{8}$.

\section{Polarimeter Model}

We consider a polarimeter that consists of an AHWP that rotates at a frequency $f_{0}$, a linear polarizer, and a power detector (e.g., a bolometer), as shown in Fig. 1. The angle $\rho$ gives the rotation angle of the AHWP around its axis of symmetry $z$. In such a polarimeter, information about the incident polarization is contained in the intensity that is detected by the detector as a function of $\rho$. To a good approximation, the detected intensity is sinusoidal as a function of $\rho$ with a frequency of $4 f_{0}$ when there is a high signal-to-noise ratio. Our primary interest in this paper is to analyze the detected intensity as a function of $\rho$, which we call IVA (intensity versus angle), with the purpose of reconstructing the incident polarization.

We make a number of simplifying assumptions in order to focus attention on the main physical effects. All calculations assume normal incidence. Throughout the paper the incident polarization is assumed linear because the polarization of the CMB is not expected to be elliptical. The calculations neglect the effect of absorption by the wave plate or effects of reflections between media that have different indices of refraction. Some of the interference phenomena that occur due to multiple reflections in the stack tend to average with a wide detection bandwidth. Also, both differential reflection and absorption effects appear primary at $2 f_{0}$ and are thus separable from the main signal at or near $4 f_{0}$ when the AHWP is rotated continuously.

Consider an input Stokes vector $\mathbf{S}_{\text {in }}$ of radiation propagating along the $z$ axis that is incident on the polarimeter. The Stokes vector incident on the detector, which we call the output Stokes vector, is

$$
\begin{aligned}
\mathbf{S}_{\text {out }}= & G \prod_{i=1}^{m}\left[R\left(-\rho-\theta_{i}\right) \Gamma_{i}(\Delta \delta) R\left(\rho+\theta_{i}\right)\right] \\
& \times \mathbf{S}_{\text {in }}\left(\nu, \alpha_{\text {in }}, P_{\text {in }}\right)
\end{aligned}
$$

where

$$
\begin{gathered}
\Delta \delta=2 \pi \frac{\nu}{c}\left|n_{o}-n_{e}\right| d, \\
\mathbf{S}_{\text {in }}=\left(I_{\text {in }}, Q_{\text {in }}, U_{\text {in }}, 0\right) \\
=I(\nu)\left(1, P_{\text {in }} \cos 2 \alpha_{\text {in }}, P_{\text {in }} \sin 2 \alpha_{\text {in }}, 0\right),
\end{gathered}
$$

$G$ is the Mueller matrix of the linear polarizer, $R$ is a rotation matrix, $\Gamma$ is the Mueller matrix of a retarder, and $\mathbf{S}_{\text {in }}$ is the Stokes vector of the incident radiation, which is a function of the electromagnetic frequency, the polarization angle $\alpha_{\text {in }}$, and the degree of polarization $P_{\text {in }}$. Information about the spectrum of the incident radiation is contained in $I(\nu)$. We initially assume that the intensity of the incident radiation is constant with frequency, $I(\nu)=I_{0}=$ const. We discuss the effects of a nonconstant incident spectrum in Section 5. Equation (1) assumes $m$ wave plates in the 
stack; in this paper $m=1,3$, or 5 . The variable $\Delta \delta$ is the retardance of a single wave plate and is a function of the ordinary and extraordinary indices of refraction $n_{o}$ and $n_{e}$, respectively, the thickness of a single wave plate $d$, and the electromagnetic frequency of light $\nu$. We also assume that $\alpha_{\text {in }}$ and $P_{\text {in }}$ are independent of $\nu$. The components of the Mueller matrices are

$$
\begin{gathered}
\Gamma(\Delta \delta)=\left[\begin{array}{cccc}
1 & 0 & 0 & 0 \\
0 & 1 & 0 & 0 \\
0 & 0 & \cos \Delta \delta & -\sin \Delta \delta \\
0 & 0 & \sin \Delta \delta & \cos \Delta \delta
\end{array}\right], \\
R(\psi)=\left[\begin{array}{cccc}
1 & 0 & 0 & 0 \\
0 & \cos 2 \psi & -\sin 2 \psi & 0 \\
0 & \sin 2 \psi & \cos 2 \psi & 0 \\
0 & 0 & 0 & 1
\end{array}\right], \\
G=\frac{1}{2}\left[\begin{array}{cccc}
1 & 1 & 0 & 0 \\
1 & 1 & 0 & 0 \\
0 & 0 & 0 & 0 \\
0 & 0 & 0 & 0
\end{array}\right] .
\end{gathered}
$$

As shown in Fig. 1, we choose the transmission axis of the ideal linear polarizer to be aligned with the $+x$ axis. We define all the angles of rotation about the $z$ axis with respect to the transmission axis of the grid. According to the usual convention, angles increase in the counterclockwise direction from the $+x$ axis in the $x y$ plane. The relative orientation of plate $i=$ $2,3 \ldots$ in the stack relative to the first plate is given by $\theta_{i}$. The ordinary axis of the first plate is aligned with the $x$ axis when $\rho=0$ degrees. We use the notation $\theta$ to denote the entire set of relative orientation angles.

The output of the detector is a function of its detection bandwidth and the intensity term of $\mathbf{S}_{\text {out }}$. We assume that the detector has top-hat response of width $\Delta \nu$ about a center frequency $\nu_{c}$. The limit $\Delta \nu \rightarrow 0$ is equivalent to illuminating the polarimeter with monochromatic light. With these assumptions the first element of the output Stokes vector can be written as

$$
\begin{aligned}
\left\langle I_{\text {out }}\right\rangle\left(\nu_{c}, \Delta \nu, \alpha_{\text {in }}, P_{\text {in }}, \boldsymbol{\theta}, \rho\right)= & \int_{\nu_{c}-\frac{\Delta \nu}{2}}^{\nu_{c}+\frac{\Delta \nu}{2}} I_{\text {out }}\left(\nu, \alpha_{\text {in }}, P_{\text {in }}, \boldsymbol{\theta}, \rho\right) \\
& \times \mathrm{d} \nu .
\end{aligned}
$$

A plot of $\left\langle I_{\text {out }}\right\rangle$ as a function of $\rho$ is the IVA. (Throughout this paper angle brackets \langle\rangle denote integration over frequency.) For a single HWP, $I_{\text {out }}$ of Eq. (7) is
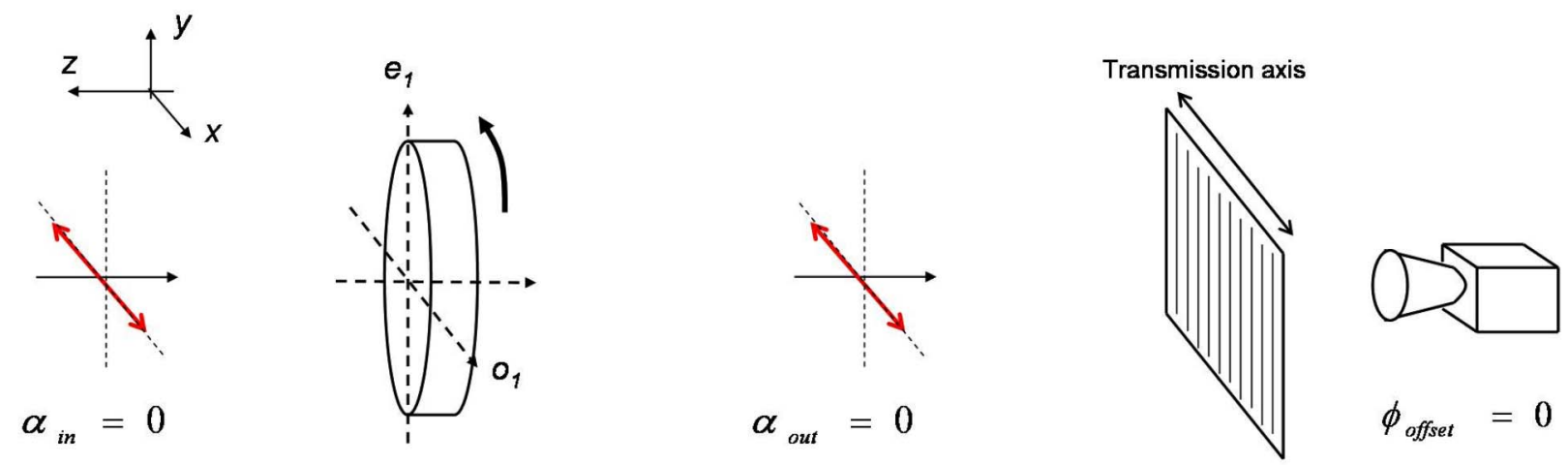

Single HWP

Linear polarizer

Power detector
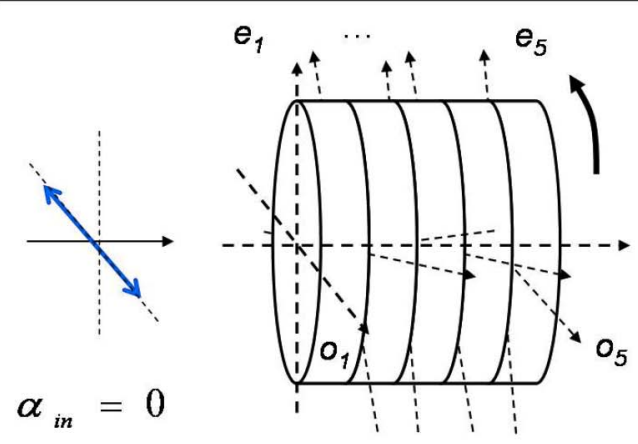

AHWP

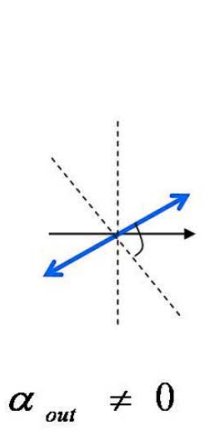

Transmission axis

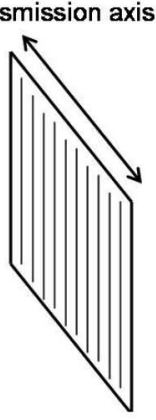

Linear polarizer

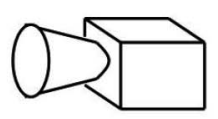

$\phi_{\text {offset }} \neq 0$

Power detector

Fig. 1. (Color online) Schematic diagram of the HWP polarimeter model. The transmission axis of a linear polarizer is parallel to the $x$ axis. 


$$
\begin{aligned}
I_{\text {out }}(\nu)= & \frac{I_{0}}{2}\left[1+P_{\text {in }} \cos 2 \alpha_{\text {in }} \cos ^{2} \frac{\Delta \delta(\nu)}{2}\right. \\
& \left.+P_{\text {in }} \sin ^{2} \frac{\Delta \delta(\nu)}{2} \cos \left(4 \rho-2 \alpha_{\text {in }}\right)\right],
\end{aligned}
$$

and an analytic integration over any bandwidth is straightforward. For a three- and a five-stack AHWP, the analytic expressions are more complicated.

One of the goals of this paper is to discuss quantitatively how the amplitude and phase of the IVA depend on the construction parameters of the AHWP. Specifically, we make a quantitative mapping between the measured amplitude and phase of the IVA and the two parameters characterizing the incident polarization, the degree of input polarization $P_{\text {in }}$, and its orientation angle $\alpha_{\text {in }}$.

When there is a finite detection bandwidth $\Delta \nu \neq 0$, the amplitude and phase of the IVA are calculated in the following way. We calculate the intensities as a function of angle $\rho$ for each frequency within the bandwidth. We then sum the calculated intensities angle by angle to obtain a final IVA. The amplitude and phase are determined from that IVA.

The analysis described in Sections $\underline{4}-\underline{6}$ are computational. IVAs have been calculated as $\bar{a}$ function of various parameters of the incident radiation and of the construction of the HWP. Many of the results were calculated by two independent computer codes to check for errors. Where practical, the results were compared to analytical calculations, and agreement has been verified.

\section{Figures of Merit}

Figure 2 shows the IVA for the case of a single sapphire HWP and for an AHWP made of a stack of three and five sapphire plates. Table 1 lists the parameters of the plates used to generate these IVAs. The thickness of each wave plate gives $\Delta \delta=\pi$ when $\nu_{\mathrm{WP}}=$ $300 \mathrm{GHz}$. The top panels in Fig. 2 show the IVA for a monochromatic detection bandwidth $(\Delta \nu=0)$. In the panels on the bottom, the detection bandwidth is $\Delta \nu=60 \mathrm{GHz}$. The incident light is polarized parallel to the transmission axis of the grid, $\alpha_{\mathrm{in}}=0$ degrees.

Several generic features are apparent. The reduction in the amplitude of the IVA with a single HWP (left column) is a consequence of its chromaticity. Linear input polarization becomes elliptical when it passes through a wave plate that is optimized for a different frequency. There is substantially smaller reduction in amplitude of modulation for the three-stack (middle column) or five-stack (right column) AHWPs. However, whereas for a single plate the phase of the IVA is the same between different frequencies, or with a broad detection bandwidth, it becomes a function of frequency for the case of an AHWP. We define the phase angle $\phi$ of the IVA as

$$
\left\langle I_{\text {out }}\right\rangle=A_{0}+A_{4} \cos (4 \rho-4 \phi),
$$
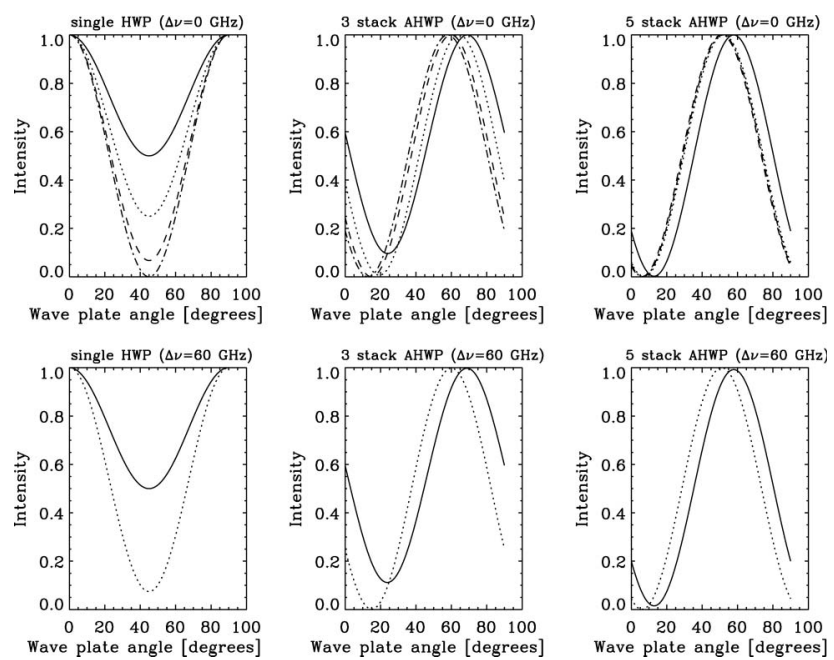

Fig. 2. IVA for monochromatic light (top panels) and for broadband radiation (bottom panels) for a single HWP, a three-stack AHWP, and a five-stack AHWP (left to right). See Table 1 for the parameters of the plates and for details about the simulations used for the calculations. Frequencies of 150 (solid), 200 (dash), 250 (dot), and 300 (dash-dot) GHz are used for the case of monochromatic light. For the broadband case, we use $150 \pm 30 \mathrm{GHz}$ (solid) and $250 \pm 30 \mathrm{GHz}$ (dot). In all the panels, the maximum intensity is normalized to 1 .

where $A_{0}$ and $A_{4}$ denote the average level and the modulation amplitude of the IVA, respectively. For the case of a single HWP the forms of $A_{0}$ and $A_{4}$ are

$$
\begin{gathered}
A_{0}=\frac{I_{0}}{2}\left(\Delta \nu+P_{\text {in }} \cos 2 \alpha_{\text {in }} \int_{\nu_{c}-\frac{\Delta \nu}{2}}^{\nu_{c}+\frac{\Delta \nu}{2}} \cos ^{2} \frac{\Delta \delta(\nu)}{2} \mathrm{~d} \nu\right), \\
A_{4}=\frac{I_{0} P_{\text {in }}}{2} \int_{\nu_{c}-\frac{\Delta \nu}{2}}^{\nu_{c}+\frac{\Delta \nu}{2}} \sin ^{2} \frac{\Delta \delta(\nu)}{2} \mathrm{~d} \nu
\end{gathered}
$$

From a comparison of Eqs. (8) and (9), it is evident that in this case $\phi=\alpha_{\text {in }} / 2$ and that it is independent of frequency. Since we chose $\alpha_{\text {in }}=0$ degrees for the simulation shown in the left panels of Fig. $2, \phi=0$ degrees. However, for an AHWP, the phase $\phi$ is a function of the thickness of the HWP, the detection bandwidth, and the relative orientation angles. Mathematically $\phi=\phi\left(\nu_{c}, \Delta \nu, \alpha_{\mathrm{in}}, \boldsymbol{\theta}\right)$, and therefore the IVAs in the middle and right columns of Fig. 2 show nonzero phase angles. We define this overall "phase offset" of the three- and five-stack AHWPs as $\phi_{0}$. The quantity $\phi_{0}$ is the value of $\phi$ when $\alpha_{\text {in }}=$ 0 degrees (e.g., $\phi_{0} \sim 65$ degrees for the solid line of the middle bottom panel of Fig. 2).

A useful figure of merit for the operation of a polarimeter is the "modulation efficiency" [19], defined as

$$
\epsilon=\epsilon\left(\nu_{c}, \Delta \nu, \alpha_{\text {in }}, P_{\text {in }}, \boldsymbol{\theta}\right)=\frac{P_{\text {out }}}{P_{\text {in }}} .
$$

The efficiency $\epsilon$ is a measure of the depolarization introduced by the polarimeter and is an essential 
Incident intensity

Indices of refraction of sapphire ${ }^{a}$

Thickness of each wave plate, $d$

Bandwidth of frequency, $\nu_{c}+\Delta \nu$

Orientation angles of three-stack AHWP, $\boldsymbol{\theta}_{3}$

Orientation angles of five-stack AHWP, $\boldsymbol{\theta}_{5}$

Resolution of frequency

Resolution of wave plate angle
$I=1$

$n_{o}=3.047, n_{e}=3.364$

$1.58 \mathrm{~mm}\left(\leftrightarrow \nu_{\mathrm{WP}}=300 \mathrm{GHz}\right)$

$150 \pm 30 \mathrm{GHz}, 250 \pm 30 \mathrm{GHz}$

$(0,58,0)$ degrees

$(0,29,94.5,29,2)$ degrees

$0.5 \mathrm{GHz}$

0.1 degree

${ }^{a}$ The sapphire indices at $1.5 \mathrm{~K}$ are extrapolated in frequency from Loewenstein $[\underline{23}, \underline{24}]$.

element in reconstructing the incident polarization $P_{\text {in }}$ from the one measured by the experiment $P_{\text {out }}$. In this paper we calculate $\epsilon$ by extracting $P_{\text {out }}$ from the IVA. $P_{\text {out }}$ is calculated from the ratio,

$$
P_{\text {out }}=P_{\text {out }}\left(\nu_{c}, \Delta \nu, \alpha_{\text {in }}, P_{\text {in }}, \boldsymbol{\theta}\right)=\frac{\left\langle I_{\text {out }}\right\rangle_{\max }-\left\langle I_{\text {out }}\right\rangle_{\text {min }}}{\left\langle I_{\text {out }}\right\rangle_{\max }+\left\langle I_{\text {out }}\right\rangle_{\min }} .
$$

Here $\left\langle I_{\text {out }}\right\rangle_{\max }$ and $\left\langle I_{\text {out }}\right\rangle_{\min }$ are the maximum and minimum of the IVA for angles $0 \leq \rho<90$. (The modulation efficiency that was calculated in our earlier publication [20] assumed a somewhat different and less accurate functional form for $P_{\text {out }}$. See Section $\underline{6}$ for more details.) Using Eqs. (8), (10), and (11), it is straightforward to show that for a single HWP and a single frequency

$$
P_{\text {out }}(\nu)=\frac{P_{\text {in }} \sin ^{2} \frac{\Delta \delta(\nu)}{2}}{1+P_{\text {in }} \cos 2 \alpha_{\text {in }} \cos ^{2} \frac{\Delta \delta(\nu)}{2}},
$$

and therefore

$$
\epsilon(\nu)=\frac{P_{\text {out }}}{P_{\text {in }}}=\frac{\sin ^{2} \frac{\Delta \delta(\nu)}{2}}{1+P_{\text {in }} \cos 2 \alpha_{\text {in }} \cos ^{2} \frac{\Delta \delta(\nu)}{2}} .
$$

There are two cases for which this expression is particularly useful, (i) when $P_{\text {in }}$ is sufficiently small such that the denominator is approximately 1, and (ii) when $\alpha_{\text {in }}=45$ degrees (for any level of $P_{\text {in }}$ ). In both of these cases

$$
\epsilon(\nu)=\sin ^{2} \frac{\Delta \delta(\nu)}{2},
$$

and $\epsilon$ is only a function of the retardance of the HWP and is independent of $P_{\text {in }}$. In the first case $\epsilon$ also does not depend on $\alpha_{\text {in }}$.

We note that instead of using Eq. (13), a more generally appropriate process for extracting the modulation efficiency is by fitting the IVA to a harmonic series of sine waves and then calculating

$$
\epsilon=\frac{A_{4}}{A_{0}}
$$

where $A_{0}$ and $A_{4}$ are the coefficients of the zeroth and fourth harmonic terms, respectively. For the results presented in this paper, there is no difference between the two processes.

The upper panels in Fig. 3 give the modulation efficiency of a single HWP and of a three- and a fivestack AHWP as a function of frequency. To calculate these efficiencies, we analyzed monochromatic IVAs. The reduction in efficiency for the three-stack AHWP at a frequency of, for example, $150 \mathrm{GHz}$ can be mapped to the smaller amplitude IVA in Fig. 2 for the same frequency. The set of three panels shows that a larger number of plates in the stack gives a broader bandwidth of high modulation efficiency.

In order to reconstruct the polarization angle $\alpha_{\text {in }}$ of the incident polarization, it is essential to examine the phase angle of the IVA. The lower panels in Fig. 3 show $\phi_{0}$ as a function of frequency. They were also extracted from IVAs calculated with monochromatic detection bandwidths. The phase offset varies with frequency even near the center frequency $\nu_{\mathrm{WP}}$.

To gain some insight into the effects that we quantify in subsequent sections, let us compare the middle panels of Figs. 2 and 3 in more detail. The solid lines shown in Fig. $\overline{2}$ were calculated for a center frequency of $150 \mathrm{GHz}$. The upper panel in Fig. 2 shows the corresponding monochromatic IVA. The modulation efficiency of $\sim 0.95$ and phase angle of $\sim 70$ degrees of that IVA can be read directly from the
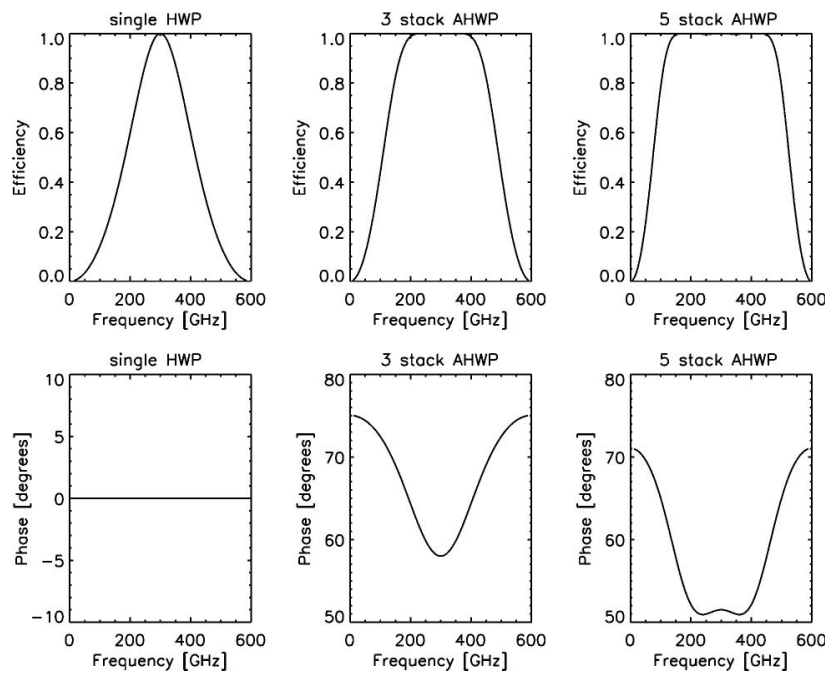

Fig. 3. Modulation efficiency $\epsilon=\epsilon\left(\nu, \Delta \nu=0, \alpha_{\text {in }}=0, \boldsymbol{\theta}\right)$ (top) and the phase offset $\phi_{0}=\phi\left(\alpha_{\text {in }}=0, \nu, \Delta \nu=0, \boldsymbol{\theta}\right)$ (bottom) for the single HWP (left) and the three- (middle) and five-stack (right) AHWPs as a function of frequency. 
middle panels of Fig. 3 at a frequency of $150 \mathrm{GHz}$. The lower panel of Fig. $\overline{2}$ shows an IVA that has been calculated for a detection bandwidth of $\pm 30 \mathrm{GHz}$ around $150 \mathrm{GHz}$. It was calculated by averaging the intensities at different frequencies. We used a frequency resolution of $0.5 \mathrm{GHz}$ (see Table 1). Each of these IVAs has a modulation efficiency and phase offset that can be read off from the middle panels of Fig. 3. Both the modulation efficiency and the phase offset vary over the bandwidth. As a consequence, the resulting IVA is a superposition of sine waves with different amplitudes and phases. Hence the final IVA is also a sine wave, but its amplitude and phase depend on averaging intensities over frequencies.

In the next section we discuss how the efficiency $\epsilon$ and the phase angle $\phi$ depend on the center frequency and the detection bandwidth, and how to relate them to the parameters of the incident polarization $P_{\text {in }}$ and $\alpha_{\text {in }}$.

\section{Reconstruction of $P_{\text {in }}$ and $\alpha_{\text {in }}$}

\section{A. Modulation Efficiency and Phase}

The top panels of Fig. 4 show the modulation efficiency of a single HWP, three-stack, and five-stack AHWPs as a function of bandwidth around $\nu_{c}=300 \mathrm{GHz}$. The different curves correspond to different input polarization angles $\alpha_{\text {in }}$. To calculate the modulation efficiency, we used $P_{\text {in }}=1$. For $\alpha_{\text {in }}=0$ degrees, modulation efficiency that is larger than 0.99 is achieved with a bandwidth of $200(300) \mathrm{GHz}$ for the three- or five-stack AHWP, while a single HWP achieves a bandwidth of only $50 \mathrm{GHz}$. For a given bandwidth, the modulation efficiency is a function of the orientation of the incident polarization $\alpha_{\text {in }}$. So in order to reconstruct $P_{\text {in }}$, informa-
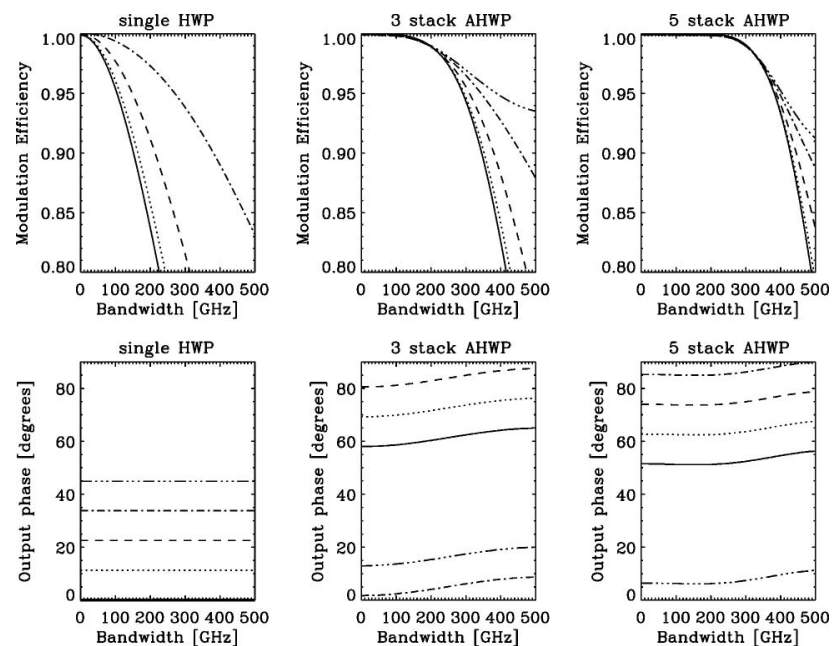

Fig. 4. Top: Modulation efficiency of the single HWP and the three- and the five-stack AHWPs as a function of detection bandwidth for input polarization angle of 0 (solid line), 22.5 (dot), 45 (dash), 67.5 (dot-dash), and 90 (three-dot dash) degrees. Bottom: Output phase angle of the single, three-, and five-stack AHWPs as a function of detection bandwidth for the same input polarization angles as the top panels. For both the modulation efficiency and the phase, $\nu_{c}=\nu_{\mathrm{WP}}$. tion about $\alpha_{\text {in }}$ needs to be extracted first from the measured phase angle $\phi$.

The bottom panels of Fig. 4 show the output phase angle $\phi$ as a function of bandwidth around $\nu_{c}=300 \mathrm{GHz}$. The phase of the single HWP shows a flat response over the bandwidth. The phases of the IVA of the three- and five-stack AHWPs are a function of bandwidth, a result consistent with the bottom row of Fig. 3. For a given bandwidth the phase angle $\phi$ has an overall offset $\phi_{0}$.

The conclusions so far are that, if the incident radiation is known to be fully polarized and the detection bandwidth is known, then the orientation angle of the incident polarization and the modulation efficiency can be extracted. Alternatively, if the orientation angle of the incident fully polarized radiation is known, then the modulation efficiency and an equivalent detection bandwidth can be extracted. These situations are encountered in the laboratory when calibrating the polarimeter.

\section{B. $\boldsymbol{P}_{\text {out }}$ versus $\boldsymbol{P}_{\text {in }}$}

The results in Fig. 4 were calculated with the assumption of incident polarization $P_{\text {in }}=1$. We relax this assumption in Fig. 5 , which shows $P_{\text {out }}$ as a function of $P_{\text {in }}$ for various angles $\alpha_{\text {in }}$. The local slope of each curve is the modulation efficiency $\epsilon$. The modulation efficiency is a function of both $\alpha_{\text {in }}$ and $P_{\text {in }}$. That this is the case for a single HWP is evident from Eq. (15). In an actual observation, both $P_{\text {in }}$ and $\alpha_{\text {in }}$ are $a$ priori unknown, which suggests that reconstructing the polarimeter modulation efficiency, or the incident polarization $P_{\text {in }}$, is subject to additional uncertainty. In many practical cases, this is not a limitation for reasons that we now discuss.
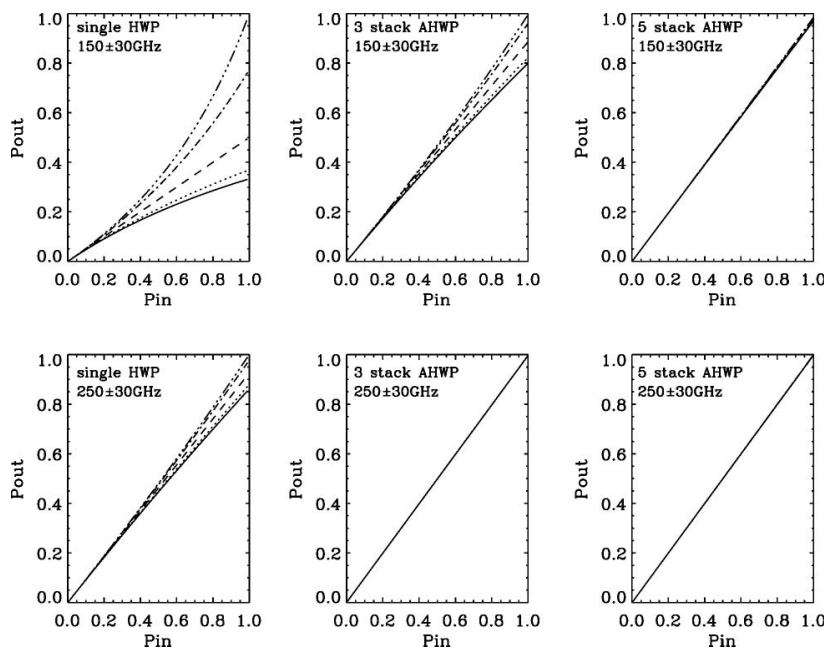

Fig. 5. Extracted degree of polarization $P_{\text {out }}$ as a function of the degree of polarization of the incident light $P_{\text {in }}$ for the single-, three, and five-stack AHWPs. Each curve corresponds to the input polarization angle of 0 (solid line), 22.5 (dot), 45 (dash), 67.5 (dotdash), and 90 (three-dot dash) degrees. The frequency and the bandwidth are $\nu_{c} \pm \Delta \nu=150 \pm 30 \mathrm{GHz}$ (top) and $250 \pm 30 \mathrm{GHz}$ (bottom). For all the panels, $\nu_{\mathrm{WP}}=300 \mathrm{GHz}$. 
Figure 5 shows that, in cases where the incident polarization is known to be small, the modulation efficiency is to a good approximation constant and does not depend either on $P_{\text {in }}$ or on $\alpha_{\text {in }}$ (for the simple case of a single HWP, see Eqs. (15) and (16)). The region where the approximation "small incident polarization" is valid depends on the construction parameters of the HWP and the detection bandwidth. For example, for the top middle and bottom left panels, the approximation is valid for $P_{\text {in }} \lesssim 0.2$. It is valid for a much larger range of $P_{\text {in }}$ when using a five-stack (see right panels), and even for the three-stack AHWP when it is used reasonably close to the designed band center (see middle bottom panel).

We note that the HWPs that are used for the calculations shown in Fig. 5 are each designed for a center frequency of $300 \mathrm{GHz}$ (see Table 1). Therefore the top left panel that shows the largest variation of the modulation efficiency with incidence angle is never likely to be used in practice. It describes a single HWP optimized for $300 \mathrm{GHz}$ that is used for a band around $150 \mathrm{GHz}$. This panel is only shown for didactic purposes.

We find then that in many practical situations there is a unique relation between $P_{\text {in }}$ and $P_{\text {out }}$, a relation that does not depend on the orientation angle of $\alpha_{\text {in }}$. In the more general case when the value of $P_{\text {out }}$ depends both on $P_{\text {in }}$ and on $\alpha_{\text {in }}$, the value of $\alpha_{\text {in }}$ needs to be determined first from the IVA. This is straightforward for a single HWP because the phase $\phi$ of the IVA is equal to $\alpha_{\text {in }} / 2$ for any detection bandwidth; see, for example, the bottom left panel of Fig. 4. The case of an AHWP is discussed in Subsection 4. $\bar{C}$, but the conclusion is that, for a specified bandwidth, there is a unique relationship between the phase $\phi$ and the angle $\alpha_{\text {in }}$. Therefore the procedure for finding $P_{\text {in }}$ is to first determine $\alpha_{\text {in }}$ using the phase of the IVA and then to use the relation between $P_{\text {out }}$ and $P_{\text {in }}$ that is appropriate for this $\alpha_{\text {in }}$.

Laboratory measurements of modulation efficiency typically use incident polarizations that are close to $P_{\text {in }}=1$ in order to increase the signal-to-noise ratio of the measurement. Figure 5 demonstrates that determinations of $\epsilon$ depend on the polarization angle $\alpha_{\text {in }}$. An efficiency value that was determined in the laboratory using a particular angle $\alpha_{\text {in }}$ will not in general correspond to the modulation efficiency of the polarimeter during actual observations for which $\alpha_{\text {in }}$ is not known. A simple remedy is to align the incident polarization in the laboratory such that $\alpha_{\text {in }}=$ 45 degrees. For that particular value, the efficiency $\epsilon_{45}$ is a constant as a function of $P_{\text {in }}$ and is equal to the same efficiency that would be measured with small incident polarizations. Table 2 summarizes this point in a quantitative way. The values shown give the efficiency expected with $\alpha_{\text {in }}=45$ degrees for different frequency bands and for different HWP configurations. The upper and lower values marked with \pm give the additional increments of efficiency that would be determined if $\alpha_{\text {in }}$ was 90 (for plus) or 0 (for minus) degrees. For example, the mod-
Table 2. Modulation Efficiency at $P_{\text {in }}=0.1$ with $\alpha_{\text {in }}=45$ Degrees ${ }^{a}$

\begin{tabular}{lcc}
\hline & $150 \pm 30 \mathrm{GHz}$ & $250 \pm 30 \mathrm{GHz}$ \\
Single HWP & $0.50_{-0.045}^{+0.055}$ & $0.93_{-0.015}^{+0.015}$ \\
Three-stack AHWP & $0.89_{-0.02}^{+0.02}$ & $0.996_{-0.000}^{+0.001}$ \\
Five-stack AHWP & $0.976_{-0.001}^{+0.002}$ & $0.999_{-0.000}^{+0.001}$ \\
\hline
\end{tabular}

${ }^{a}$ The modulation efficiency is calculated as a slope of the $P_{\text {out }}-$ $P_{\text {in }}$ relationship in Fig. 5 . The quoted errors are $\epsilon_{\max }-\epsilon_{45}$ and $\epsilon_{\min }-\epsilon_{45}$, where $\epsilon_{45}$ corresponds to the modulation efficiency at $\alpha_{\text {in }}=45$ degrees at $P_{\text {in }}=0.1$. The maximum and the minimum modulation efficiencies correspond to $\alpha_{\text {in }}=90$ and 0 degrees, respectively.

ulation efficiency of a single HWP (that is, constructed according to the parameters given in Table 1) at $150 \mathrm{GHz}$ with a bandwidth of $\pm 30 \mathrm{GHz}$ is 0.5 when measured with $\alpha_{\text {in }}=45$ degrees. This value of $\epsilon$ does not depend on the magnitude of $P_{\text {in }}$. Yet for observations with $P_{\text {in }}=0.1$ the modulation efficiency would be $0.055(0.045)$ for $\alpha_{\text {in }}=90(0)$ degrees. Whereas for the single HWP, the variation in modulation efficiency could be as large as $10 \%$; it is about $2 \%$ or smaller with the three- or five-stack AHWP. These values depend on the construction parameters of the HWP and on the detection bandwidth and thus cannot be taken as general.

\section{C. $\phi$ versus $\alpha_{\text {in }}$}

In Subsection 4.B, we investigated how the measured degree of polarization $P_{\text {out }}$ relates to the input polarization $P_{\text {in }}$. We now quantify a similar relationship between $\phi$ and $\alpha_{\text {in }}$. Figure 6 shows the phase angle as a function of the input polarization angle for the three- and five-stack AHWPs. (Recall that $\phi$ is a constant over frequency for a single HWP; see the bottom left panel of Fig. 4.) The panels show that $\alpha_{\text {in }}$ and $\phi$ have a linear relationship with a slope of 0.5 and that this slope does not depend on the
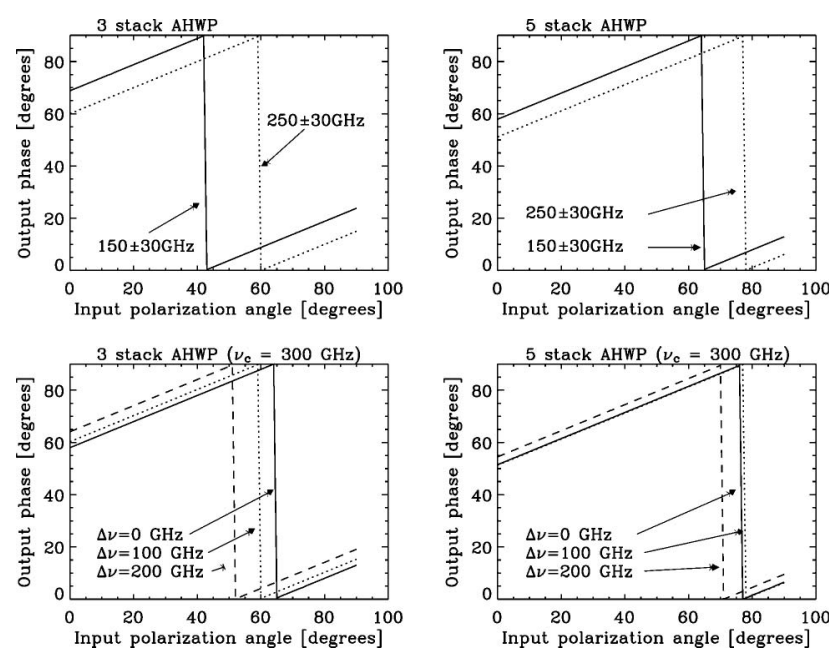

Fig. 6. Output phase angle of the three- (left) and the five-stack (right) AHWPs as a function of the input polarization angle. The top panels give results for 150 (solid) and $250 \mathrm{GHz}$ (dot), each with a fixed bandwidth of $\pm 30 \mathrm{GHz}$. The bottom panels give results for a fixed center frequency of $300 \mathrm{GHz}$ with bandwidths of \pm 0 (solid), \pm 100 (dot), and \pm 200 (dash) GHz. 
construction parameters of the HWP nor on the detection bandwidth. However, the phase offset is a function of $\nu_{c}$ and $\Delta \nu$.

The magnitude of the phase offset $\phi_{0}$ is a critical parameter in the reconstruction of an unknown incident polarization angle $\alpha_{\text {in }}$. Since this phase offset is a function of the spectral response of the instrument, it can either be calculated, if the spectral response is known, or measured in the laboratory by varying $\alpha_{\text {in }}$ of a known source and by extracting $\phi$ from the IVA. (See, however, Section 5 for important caveats.) Errors in this calibration will propagate to errors in the determination of $\alpha_{\text {in }}$ for a source whose polarization properties are not known.

The direction of rotation of the AHWP affects the relationship between $\phi$ and $\alpha_{\text {in }}$. With a single HWP, $\phi= \pm \alpha_{\text {in }} / 2$, where the sign is determined by the direction of rotation. For our particular choice of directions (see Fig. 1) we have $\phi=+\alpha_{\text {in }} / 2$. However, the orientation angles of the stack of plates break the rotational symmetry for an AHWP, and in general there are four possible choices:

$$
\begin{gathered}
\phi= \pm \frac{1}{2} \alpha_{\text {in }}+\phi_{0}, \\
\phi= \pm \frac{1}{2} \alpha_{\text {in }}+\frac{\pi}{2}-\phi_{0} .
\end{gathered}
$$

In Eq. (19) the phase offset is no longer $\phi_{0}$, but $\frac{\pi}{2}-\phi_{0}$. For our particular choice, where both $\rho$ and the AHWP orientation angles $\boldsymbol{\theta}$ are counterclockwise in the $x y$ plane (as shown in Fig. 1), Eq. (18) with a plus sign gives the relevant functional dependence.

\section{Spectrum of Incident Radiation}

So far we have assumed an incident radiation spectrum that was constant with frequency. We now address the more general case where the spectrum of the incident radiation is a function of frequency. In this case the phase offset $\phi_{0}$ depends on the details of this spectrum. Since $\phi_{0}$ is required for reconstruction of $P_{\text {in }}$ and $\alpha_{\text {in }}$, the consequence is that knowledge of the incident spectrum is also required.

To assess this effect quantitatively, we consider three distinct spectra: (1) blackbody with the temperature of the cosmic microwave background radiation, (2) blackbody with a temperature of $300 \mathrm{~K}$, and (3) galactic dust. We choose these spectra because they are relevant for calibration and for measurements of the polarization of the CMB at frequencies between 100 and $500 \mathrm{GHz}$. We assume the following spectra $I(\nu)$ :

$$
\begin{gathered}
I_{\mathrm{CMB}}(\nu)=B\left(T_{\mathrm{CMB}}, \nu\right), \\
I_{\mathrm{dust}}(\nu)=A \nu^{\gamma} B\left(T_{\mathrm{dust}}, \nu\right), \\
I_{\mathrm{lab}}(\nu)=B\left(T_{\mathrm{lab}}, \nu\right), \\
B(T, \nu)=\frac{2 \pi h}{c^{2}} \frac{\nu^{3}}{\mathrm{e}^{\frac{h}{k} B^{T}}-1},
\end{gathered}
$$

where $B$ denotes a blackbody spectrum, $T_{\mathrm{CMB}}=$ $2.73 \mathrm{~K}, A=4 \times 10^{-7}, \quad \gamma=1.75, \quad T_{\text {dust }}=18 \mathrm{~K}$, and $T_{\text {lab }}=300 \mathrm{~K}$. We assume that the fractional polarization and the polarization angle of the incident radiation do not depend on frequency. We also assume that the degree of linear polarization of the CMB, of the galactic dust, and of a $300 \mathrm{~K}$ blackbody source are $P_{\mathrm{CMB}}=1 \times 10^{-6}, \quad P_{\text {dust }}=0.1, \quad$ and $\quad P_{\text {lab }}=1$, respectively.

The calculated phase offsets of a five-stack AHWP are summarized in Table 3 . The left table shows the phase offset in units of degrees. The right table shows the difference of the phase offsets between the different spectra. The parentheses indicate the level of difference in terms of polarization angle $\alpha_{\text {in }}$.

Assume that a $300 \mathrm{~K}$ source is used in the laboratory to calibrate the phase offset and that the laboratory measurement agrees with the phase offsets given in the right hand column of the left part of Table 3. If these values are used for either CMB or dust observations, they would give rise to errors in position angle of the polarization on the sky as given in parentheses in the two right columns of the right table. The correct prescription is to validate the design of the instrument using the laboratory measurements and then use the predicted phase offsets given assumptions or measurements of the spectra of the sources. In fact, an uncertainty in the knowledge of the spectrum would give an uncertainty in the determination of $\alpha_{\text {in. }}$. The designer of a polarimeter with an AHWP should plan for this uncertainty and its mitigation during the analysis of the data.

\section{AHWP Performance Versus Orientation Angles $\boldsymbol{\theta}$}

We have already pointed out in an earlier publication that it is relatively easy to achieve a high modulation efficiency with the three- and five-stack AHWPs in

Table 3. Offset Angles with Four Different Spectra and Difference of the Offset Phase between Different Spectra ${ }^{a}$

\begin{tabular}{ccccccc}
\hline & CMB & Dust & Lab & CMB - Dust & CMB - Lab & Dust - Lab \\
\hline $150 \pm 30 \mathrm{GHz}$ & $\phi_{0}=57.86$ & 56.69 & 57.33 & $\Delta \phi=1.17\left(\Delta \alpha_{\text {in }}=2.34\right)$ & $0.53(1.06)$ & $-0.64(-1.28)$ \\
$250 \pm 30 \mathrm{GHz}$ & 51.12 & 51.16 & 51.14 & $-0.04(-0.08)$ & $-0.02(-0.04)$ & $0.02(0.04)$ \\
$420 \pm 30 \mathrm{GHz}$ & 53.85 & 54.50 & 54.49 & $-0.65(-1.3)$ & $-0.64(-1.28)$ & $0.01(0.02)$ \\
\hline
\end{tabular}

\footnotetext{
${ }^{a}$ Each number in parentheses is the difference in terms of the polarization angle $\alpha_{\text {in }}$ on the sky. The unit of the phase is in degrees.
} 
terms of the requirements on the relative orientation of the plates [20]. In this section we expand on our earlier work and give a more thorough discussion. All of the analysis in this section assumes a constant spectrum for the incident radiation.

The left panels of Figs. 7-10 give contour plots for the modulation efficiency as a function of the orientation of the plates in the stacks. The modulation efficiency is calculated based on Eq. (13) with Eqs. (7) and (12). The right panels of the same figures show the phase offset as defined in Eq. (9).

For a three-stack AHWP, Title [18] showed that the highest modulation efficiency is achieved with a set of angle $\boldsymbol{\theta}=(0,58,0)$. The left panel of Fig. 7 shows that this modulation is a weak function of the orientation of the middle plate near peak efficiency. The right panel of Fig. 7 shows that for a second plate orientation, $80 \lesssim \theta_{2} \lesssim 100$ degrees, the phase offset $\phi_{0}$ is essentially independent of detection bandwidth. This orientation angle, however, does not give the broadest range of frequencies for high modulation efficiency. On the other hand, with an angle of 58 degrees, which gives the broadest range of modulation efficiency, the phase offset has stronger dependence on the detection bandwidth. Experiment designers need to consider this trade-off between bandwidth for high modulation and for constant phase offset.

The three-stack AHWP has zero modulation efficiency at $\theta_{2}$ close to 20 and 160 degrees and detection bandwidth of $400 \mathrm{GHz}$. This is because there is a strong variation of the phase offset angle $\phi_{0}$ with frequency near these parameters. Therefore these points in the parameter space give the resultant IVA zero modulation amplitude, and correspondingly no phase can be defined as demonstrated by the singularities in the phase offset panel. The color discontinuity extending from the phase offset singularity toward bottom right is a consequence of phase offset periodicity. It is neither an artifact nor a real discon-
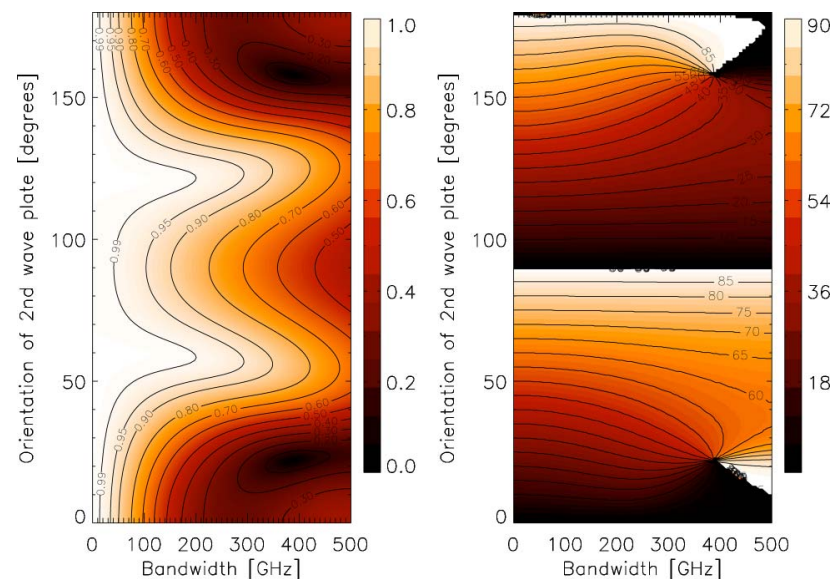

Fig. 7. (Color online) Modulation efficiency (left) and the phase offset (right) of the three-stack AHWP as a function of the angle of the second plate $\theta_{2}$ and the bandwidth $\Delta \nu$ around a center frequency of $\nu_{\mathrm{WP}}=300 \mathrm{GHz}$. The color scale of the phase offset is in units of degrees. In both plots, the input polarization angle is $\alpha_{\text {in }}=0$.
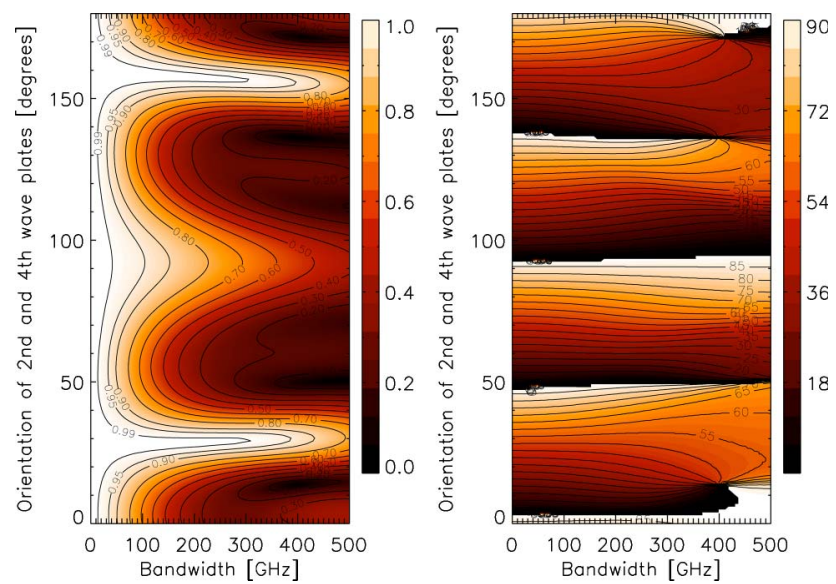

Fig. 8. (Color online) Modulation efficiency (left) and the phase offset (right) of the five-stack AHWP as a function of the orientation angles of the second and fourth plates. The other angles are fixed at the values given in Table $\underline{1}$.

tinuity. Phases that are larger than 90 degrees are interpreted as positive values close to zero. Similar features appear in Figs. 8-10.

With the five-stack $\mathrm{A} H \overline{\mathrm{W}} \overline{\mathrm{P}}$, achieving high modulation efficiency requires higher accuracy of alignment of the second, third, and fourth wave plates than that required in the case of the three-stack AHWP. Little accuracy is required from the orientation of the fifth plate in the five-stack AHWP. The efficiency is most sensitive to the orientation of the second and fourth plates, and an accuracy of 5 degrees is required to maintain efficiency higher than 0.95 over $300 \pm 150 \mathrm{GHz}$.

In a previous publication [20], we gave results for the modulation efficiency that was based on the following expression:

$$
P_{\text {out }}=\left\langle\frac{I_{\max }-I_{\min }}{I_{\max }+I_{\min }}\right\rangle
$$

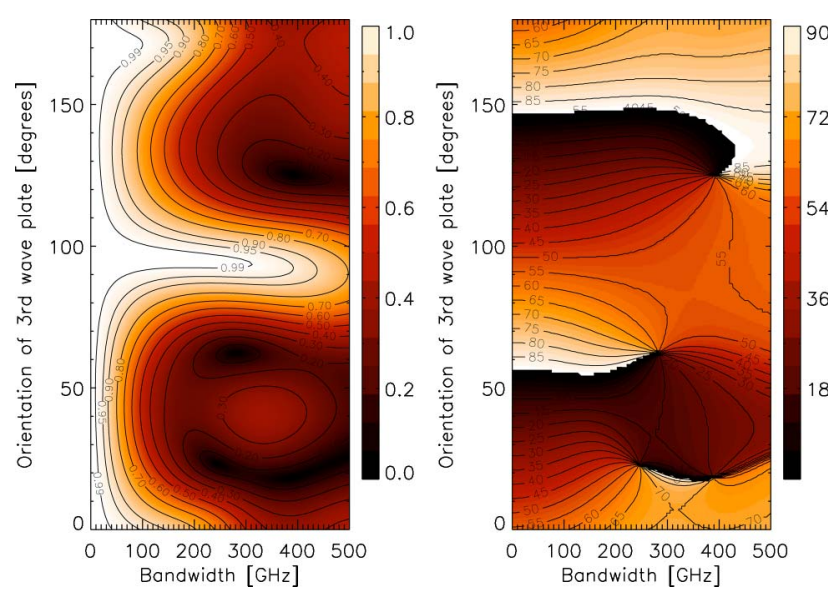

Fig. 9. (Color online) Modulation efficiency (left) and the phase offset (right) of the five-stack AHWP as a function of the orientation angle of the third plate. The other angles are fixed at the values given in Table $\underline{1}$. 

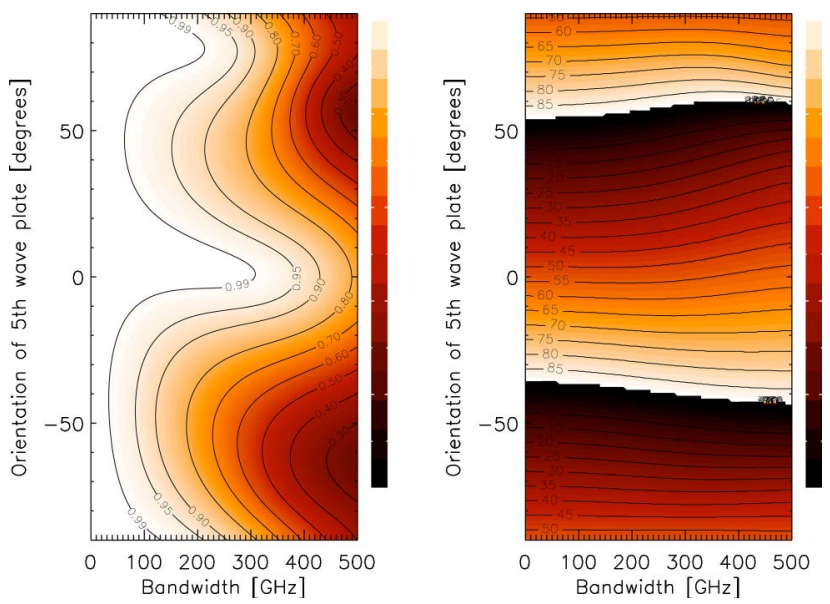

Fig. 10. (Color online) Modulation efficiency (left) and the phase offset (right) of the five-stack AHWP as a function of the orientation angle of the fifth plate. The other angles are fixed at the values given in Table $\underline{1}$.

which is different from the more correct definition given in Eq. (13). The left panel of Fig. 11 is the modulation efficiency based on Eq. (24) with the same parameters that produced Fig. 7 . The right panel shows the differences between the two results. The modulation efficiency in Fig. 7 accounts for the phase variation of the IVA curves as a function of frequency. In contrast, the modulation efficiency in Fig. 11 does not encode this variation.

\section{Experimental Data}

We measured the spectrum of a five-stack AHWP using Fourier transform spectroscopy. The five-stack AHWP was assembled using five sapphire plates and three layers of antireflection coatings on each side. It was maintained at room temperature during the measurement. Spectra were taken for three frequency bands centered at 150,250 , and $410 \mathrm{GHz}$. For brevity, we only show the results for the 150 and $410 \mathrm{GHz}$ bands. For each band, the AHWP was rotated to find the angle at which transmission
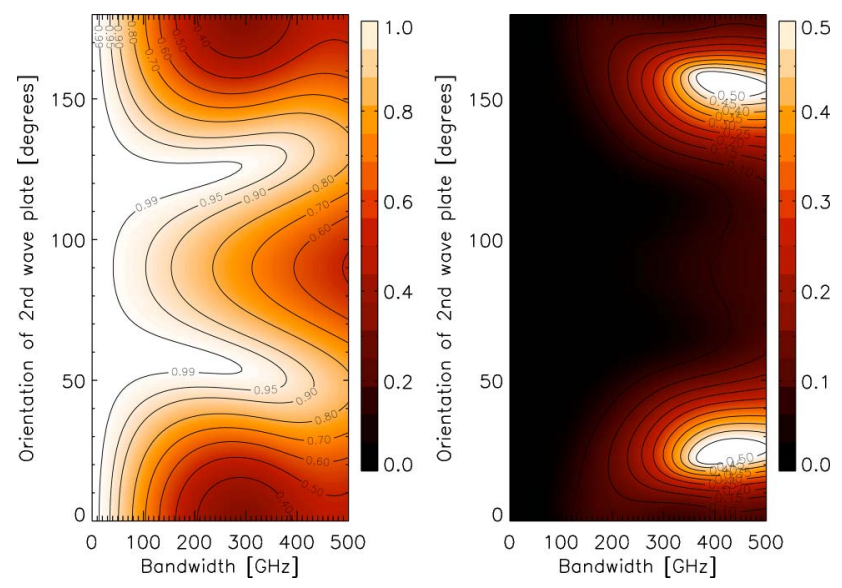

Fig. 11. (Color online) Modulation efficiency of a three-stack AHWP based on Eq, (24) (left) and the difference between this efficiency and the one calculated in Fig. $\underline{7}$ (right).

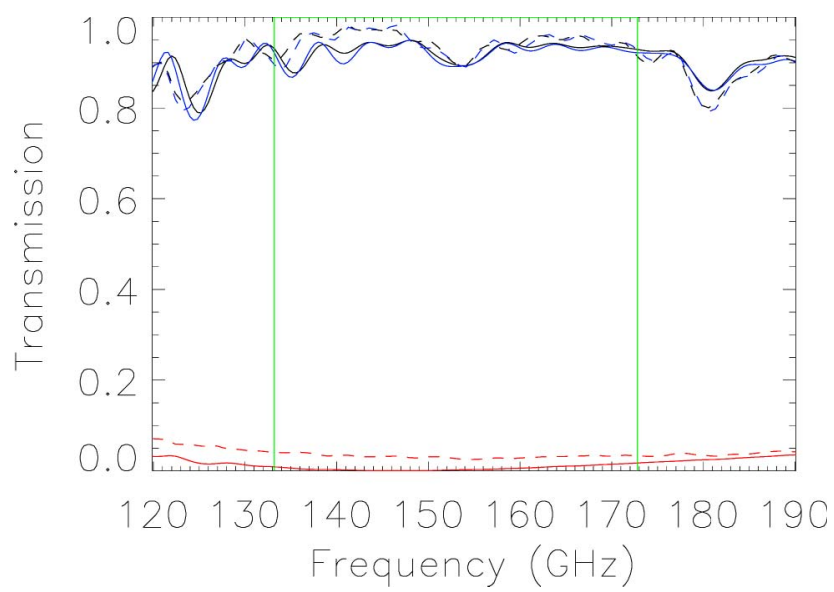

Fig. 12. (Color online) Transmission spectra (dash) and fits (solid) for the EBEX five-stack AHWP near $150 \mathrm{GHz}$. The measurements were done at an AHWP rotational position that maximized the signal at $150 \mathrm{GHz}$ (black), and at 45 (red) and 90 degrees (blue) from that angle. The text describes which construction parameters were used in the fit. The design bandwidth is bracketed by two vertical lines.

was maximized. Spectra were taken at this maximum rotational position as well as at 45 and 90 degrees from that position. The 45 degree measurement should give a minimum in the transmission, and the 90 degrees should give a second maximum. Figures 12 and 13 give the results. The transmission at $410 \overline{\mathrm{GH} z}$ is substantially lower compared to $150 \mathrm{GHz}$ because the absorption increases as a function of frequency. (For the EBEX implementation, the AHWP is maintained at $4 \mathrm{~K}$, and hence the expected transmission at $410 \mathrm{GHz}$ is expected to be $90.3 \%$ ).

The measured spectra (dashed lines) were fit (solid lines) with a model that included the orientation angles of the plates, their thicknesses, and the thicknesses of the antireflection coatings [21,22]. Using the fit parameters, we calculated the expected modulation efficiency of this AHWP at $4 \mathrm{~K}$ using the sapphire indices shown in Table 1 and for the EBEX design bandwidths. We calculate efficiencies of

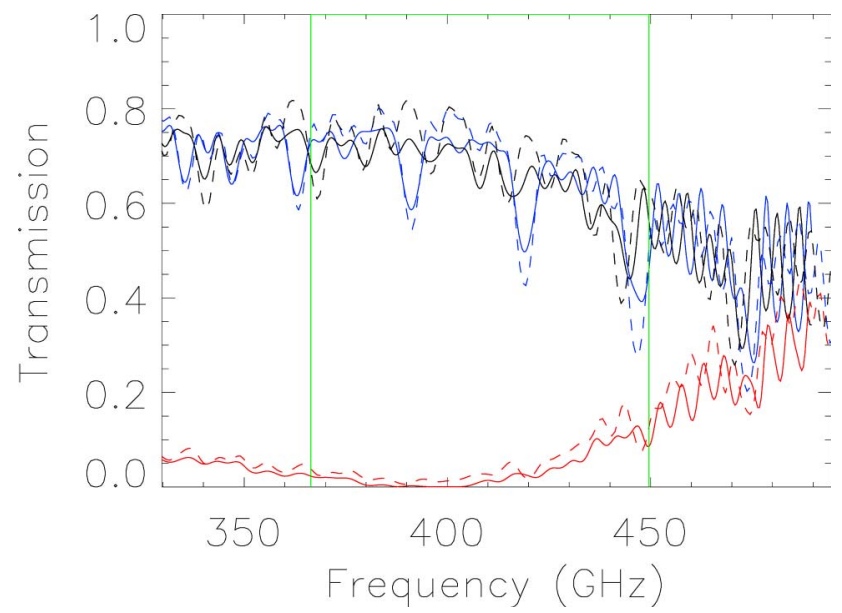

Fig. 13. (Color online) Same as Fig. 12 for a center frequency of $410 \mathrm{GHz}$. 
1., 1 , and 0.98 for $150 \pm 20,250 \pm 35$, and $410 \pm$ $42 \mathrm{GHz}$, respectively.

\section{Discussion}

We analyzed the performance of three- and five-stack AHWP polarimeters operating in the submillimeter wave band. Let us summarize the points that have been discussed and make some additional comments where appropriate.

- Three- and five-stack AHWP polarimeters provide broad bandwidth with high modulation efficiency.

- Their IVA has a phase offset that depends on the construction parameters of the stack, on the spectral response of the instrument, and on the spectrum of incident radiation. Our discussion assumed that the degree of polarization and the angle were independent of frequency with the detection bandwidth.

- If the spectral response of the instrument and the spectrum of the source are known, then measurements of the phase of the IVA can give the orientation angle of the incident polarization.

We note that in many cases, much of the radiation incident on the detector is due to emission by the telescope itself. If this emission is polarized, it too will affect the phase of the IVA and hence the measurement of the angle of incident polarization.

- Measurement uncertainties in either the spectral response of the instrument or the spectrum of the source translate to uncertainties in the reconstruction of the angle of incident polarization. The amount of uncertainty needs to be assessed on a case-by-case basis.

- Measurements of the amplitude of the IVA, which gives the degree of output polarization, can be uniquely inverted, in most cases, to give the input polarization if the modulation efficiency is known.

- In some cases, information about the angle of the incident polarization needs to be used together with the modulation efficiency to find the incident degree of polarization.

- Laboratory measurements to find the modulation efficiencies that are conducted with a source that has a known degree of polarization (typically $100 \%$ polarized radiation) should have an incident polarization angle of 45 degrees. At this angle, the measured efficiency is the same as would be measured at any angle when $P_{\text {in }}$ is small.

- We discussed how the modulation efficiency and phase offset of the polarimeters depend on errors in the orientation of the plates. Generally, an accuracy of few degrees is sufficient to ensure close to the ideal performance.

- We discussed how the incident spectrum of the radiation affects the IVA and the extraction of the parameters of the incident radiation.

- We calculated the expected modulation efficiency of the five-stack AHWP based on the experimental data. The modulation efficiencies are expected to be $\sim 1$ for 150,250 , and $410 \mathrm{GHz}$ bands and consistent with the purely calculation based results.

Our analysis assumed a spectral response of the instrument that was top-hat in shape over a range in frequencies. This is an idealization. In any practical instrument, the entire spectral response of the instrument is necessary in order to reconstruct the parameters of the incident polarization.

\section{References}

1. J. M. Kovac, E. M. Leitch, C. Pryke, J. E. Carlstrom, N. W. Halverson, and W. L. Holzapfel, "Detection of polarization in the cosmic microwave background using DASI," Nature 420, 772 (2002)

2. L. Page, G. Hinshaw, E. Komatsu, M. R. Nolta, D. N. Spergel, C. L. Bennett, C. Barnes, R. Bean, O. Dore, M. Halpern, R. S. Hill, N. Jarosik, A. Kogut, M. Limon, S. S. Meyers, N. Odegard, H. V. Peiris, G. S. Tucker, L. Verde, J. L. Weiland, E. Wollack, and E. L. Wright, "Three year Wilkinson Microwave Anisotropy Probe (WMAP) observations: polarization analysis," Astrophys. J. Suppl. Ser. 170, 335-376 (2007).

3. P. Oxley, P. Ade, C. Baccigalupi, P. deBernardis, H.-M. Cho, M. J. Devlin, S. Hanany, B. R. Johnson, T. Jones, A. T. Lee, T. Matsumura, A. D. Miller, M. Milligan, T. Renbarger, H. G. Spieler, R. Stompoer, G. S. Tucker, and M. Zaldarriaga, "The EBEX experiment," Proc. SPIE 5543, 320-331 (2004).

4. J. Kovac, BICEP/SPUD collaboration, "BICEP2 and SPUD: searching for inflation with degree-scale polarimetry from the South Pole," Bull. Am. Astron. Soc. 38, 913 (2006) .

5. A. C. Taylor, A. Challinor, D. Goldie, K. Grainge, M. E. Jones, A. N. Lasenby, S. Withington, G. Yassin, W. K. Gear, L. Piccirilo, P. Ade, P. D. Mauskopf, B. Maei, and G. Pisano, "Clover-a new instrument for measuring the b-mode polarization of the CMB," in Proceedings of the XXXVIXth Rencontres de Moriond, Exploring the Universe (2004).

6. http://quiet.uchicago.edu/.

7. T. E. Montroy, P. A. R. Ade, R. Bihary, J. J. Bock, J. R. Bond, J. Brevick, C. R. Contaldi, B. P. Crill, A. Crites, O. Dor, L. Duband, S. R. Golwala, M. Halpern, G. Hilton, W. Holmes, V. V. Hristov, K. Irwin, W. C. Jones, C. L. Kuo, A. E. Lange, C. J. MacTavish, P. Mason, J. Mulder, C. B. Nettereld, E. Pascale, J. E. Ruhl, A. Trangsrud, C. Tucker, A. Turner, and M. Viero, "Spider: a new balloon-borne experiment to measure CMB polarization on large angular scales," Proc. SPIE 6267, 62670R (2006).

8. A. Kogut, D. T. Chuss, D. Fixsen, G. F. Hinshaw, M. Limon, S. H. Moseley, N. Phillips, E. Sharp, E. J. Wollack, and K. U-Yen, "PAPPA: primordial anisotropy polarization pathfinder array," J. Opt. Soc. Am. 69, 3941 (1979).

9. http://bolo.berkeley.edu/polarbear/.

10. T. J. Jones and D. I. Klebe, "A simple infrared polarimeter," Publ. Astron. Soc. Pac. 100(9), 1158-1161 (1988).

11. S. R. Platt, R. H. Hildebrand, R. J. Pernic, J. A. Davidson, and G. Novak, "100-micron array polarimetry from the Kuiper Airborne Observatory-Instrumentation, techniques, and results," Publ. Astron. Soc. Pac. 103(11), 1193-1210 (1991).

12. R. W. Leach, D. P. Clemens, B. D. Kane, and R. Barvainis, "Polarimetric mapping of Orion using MILLIPOL-magnetic activity in BN/KL," Astrophys. J. 370, 257-262 (1991).

13. A. G. Murray, R. Nartallo, C. V. Haynes, F. Gannaway, and P. A. R. Ade, "An imaging polarimeter for SCUBA," in ESA SP-401: The Far Infrared and Submillimetre Universe (European Space Agency, 1997), p.405 . 
14. B. R. Johnson, J. Collins, M. E. Abroe, P. A. R. Ade, J. Bock, J. Borrill, A. Boscaleri, P. de Bernardis, S. Hanany, A. H. Jae, T. Jones, A. T. Lee, L. Levinson, T. Matsumura, B. Rabii, T. Renbarger, P. L. Richards, G. F. Smoot, R. Stompor, H. T. Tran, C. D. Winant, J. H. P. Wu, and J. Zuntzs, "MAXIPOL: cosmic microwave background polarimetry using a rotating half-wave plate," Astrophys. J. 665, 42-54 (2007).

15. J. H. P. Wu, J. Zuntz, M. E. Abroe, P. A. R. Ade, J. Bock, J. Borrill, J. Collins, S. Hanany, A. H. Jae, B. R. Johnson, T. Jones, A. T. Lee, T. Matsumura, B. Rabii, T. Renbarger, P. L. Richards, G. F. Smoot, R. Stompor, H. T. Tran, C. D. Winant, "MAXIPOL: data analysis and results," Astrophys. J. 665, 55-66 (2007).

16. S. Pancharatnam, "Achromatic combinations of birefringent plates," Mem. Raman Res. Inst. Bangalore 71, 137-144 (1955).

17. A. M. Title, "Improvement of birefringent filters. 2: Achromatic waveplates," Appl. Opt. 14, 445(1975).

18. A. M. Title and W. J. Rosenberg, "Achromatic retardation plates," Proc. SPIE 307, 120 (1981).
19. J. Tinbergen, Astronomical Polarimetry (Cambridge University, 1996).

20. S. Hanany, H. Hubmayr, B. R. Johnson, T. Matsumura, P. Oxley, and M. Thibodeau, "Millimeter-wave achromatic half-wave plate," Appl. Opt. 44, 4666-4670 (2005).

21. G. Pisano, G. Savini, P. A. R. Ade, V. Haynes, and W. K. Gear, "Achromatic half-wave plate for submillimeter instruments in cosmic microwave background astronomy: experimental characterization," Appl. Opt. 45, 6982-6989 (2006).

22. G. Savini, G. Pisano, and P. A. R. Ade, "Achromatic half-wave plate for submillimeter instruments in cosmic microwave background astronomy: modeling and simulation," Appl. Opt. 45, 8907-8915 (2006).

23. E. V. Loewenstein, "Optical constants for far infrared materials. I. Crystalline solids," Appl. Opt. 12, 398-406 (1973).

24. B. R. Johnson, "MAXIPOL: a bolometric, balloon-borne experiment for measuring the polarization anisotropy of the cosmic microwave background radiation," Ph.D. dissertation (University of Minnesota, 2005). 